Artículo científico

Volumen 32(1):209-223. Enero-abril, 2021

e-ISSN 2215-3608, doi:10.15517/am.v32i1.40679

http://www.revistas.ucr.ac.cr/index.php/agromeso

\title{
Variación de los precios de la naranja (Citrus sinensis L.) en México ${ }^{1}$
}

\author{
Variation in the orange (Citrus sinensis L.) prices in Mexico
}

\author{
José Alberto García-Salazar ${ }^{2}$, Fidel Bautista-Mayorga², Mercedes Borja-Bravo ${ }^{3}$,Eugenio Guzmán-Soria ${ }^{4}$
}

1 Recepción: 20 de febrero, 2020. Aceptación: 8 de julio, 2020. En este trabajo se utilizó información derivada de la tesis de maestría denominada "Planeación de la superficie sembrada y volatilidad de precios en el mercado de la naranja en México", Colegio de Postgraduados.

2 Colegio de Postgraduados, Campus Montecillo, Instituto de Socioeconomía, Estadística e Informática, Programa de Economía. Km. 36.5 Carretera México-Texcoco. Montecillo, Estado de México, CP 56230. México. jsalazar@colpos.mx (autor para correspondencia; http://orcid. org/0000-0002-9892-7618); bautista.fidel@colpos.mx (https://orcid.org/0000-0003-1480-0239)

3 Campo Experimental Pabellón-INIFAP. Km 32.5 Carretera Ags.-Zac. Pabellón de Artega, Aguascalientes. CP 20671. México. borja. mercedes@inifap.gob.mx (http://orcid.org/0000-0001-7743-6003).

4 Instituto Tecnológico de Celaya, Campus II. Departamento de Ciencias Económico-Administrativas. Av. García Cubas 1200, Esquina Ignacio Borunda. Celaya, Guanajuato. CP 38010. México. eugenio.guzman@itcelaya.edu.mx (https://orcid.org/0000-0002-4877-3403).

\section{Resumen}

Introducción. Identificar los factores que pueden aumentar los precios al productor de naranja es importante por los efectos negativos que una caída en el ingreso puede tener sobre los productores agrícolas. Objetivo. Determinar cómo la demanda de la industria y la superficie sembrada de naranja afectan los precios al productor, al consumidor y al mayoreo. Materiales y métodos. Se formuló un modelo sintético del mercado de la naranja en México que consideró la producción en superficies de riego y temporal (ciclo de lluvia), la demanda en fresco e industrial,y diferentes niveles de transmisión de precios. El modelo replicó el valor promedio de las variables más importantes en el mercado de la naranja de los años 2014-2016. Resultados. Un aumento de $5 \%$ en el consumo de la industria productora de jugo de naranja haría crecer los precios al productor en superficies de riego y bajo temporal en 11,1 y $35,8 \%$, respectivamente, y ubicaría los precios al mayoreo $31,7 \%$ por encima de los precios observados en la situación base. Una disminución de $5 \%$ en la superficie cosechada de naranja tendría un aumento de 15,2 y 49,1\% en el precio al productor en zonas de riego y temporal, con relación a la situación base. Conclusión. La superficie y la demanda industrial son medidas de control que pueden evitar la caída de los precios. Por la importancia que la producción de naranja tiene para los productores, el gobierno debería promover medidas para evitar que el aumento en la superficie de naranja provoque la disminución de los precios y lograr el compromiso de la industria de actuar a través de la demanda en situaciones de emergencia, es decir en los años de exceso de oferta de naranja.

Palabras clave: demanda, oferta, superficie sembrada, industria, modelos de simulación.

\begin{abstract}
Introduction. Identifying the factors that can increase the orange producer prices, it is important because of the negative effects that a decrease in income can have on agricultural producers. Objective. To determine how the industry demand and the area sowed with oranges affect producer, consumer, and wholesale prices. Materials
\end{abstract}


and methods. A synthetic model of the orange market in Mexico that considered the orange production in irrigated and rainfed areas (rain cycle), the fresh and industrial demand, and different levels of transmission of prices was formulated. The model replicated the average value of the most important variables in the orange market of the years 2014-2016. Results. An increase of $5 \%$ in the consumption of the orange juice producing industry would raise producer prices in irrigated areas and under seasonal conditions by 11,1 and $35,8 \%$ respectively, and would place wholesale prices $31,7 \%$ higher than the base situation. A $5 \%$ decrease in the area of orange harvested would have a 15.2 and $49.1 \%$ increase in the producer price in irrigated and seasonal areas, in relation to the base situation. Conclusion. The surface and the industrial demand are control measures which prevent the fall of prices. Due to the importance that the orange production has for the growers, the Government should promote measures to prevent the increase in the orange sowed surface from causing the fall in the price and achieve the commitment of the industry to act through demand in emergency situations, that is to say in the years of supply of orange excess.

Keywords: demand, supply, sowed surface, industry, simulation models.

\section{Introducción}

La naranja (Citrus sinensis L.) es una de las frutas más importantes en el sector agrícola de México. En el periodo 2014-2016 la superficie destinada al cultivo de la naranja fue de 336000 ha, de las cuales 25,7 \% utilizaron sistemas de riego y el 74,3\% se desarrolló en áreas de temporal (ciclo de lluvias). Con un rendimiento promedio en zonas de riego y temporal de 18,5 y 12,8 $\mathrm{t} \mathrm{ha}^{-1}$, la producción de naranja en ese periodo fue de 1,596 y 3,207 millones de toneladas (Mt), para un total de 4,802 Mt anuales (Sistema de Información Agroalimentaria y Pesquera, 2017).

En el periodo 2014-2016, el consumo nacional aparente de naranja se ubicó en 4,774 Mt, distribuido de la siguiente manera: 55,8 \% fue consumido en fresco en los hogares, 33,9\% fue utilizado por la industria citrícola para la producción de jugo, y 10,5\% se perdió en mermas (Food and Agriculture Organization, 2018). En el periodo señalado, los estados de Veracruz y Tamaulipas, generaron el 68,2 \% de la oferta nacional; otros estados importantes fueron Nuevo León con el 7,0 \% de la producción, San Luis Potosí con 6,9 \%, Puebla con 4,6 \%; Sonora con 3,2 \% y Yucatán con 2,9\% (Sistema de Información Agroalimentaria y Pesquera, 2017). Igual que la producción, el consumo en fresco está concentrado, y en el periodo 2014-2016 más de 21,4\% se ubicó en el Estado de México y en la Ciudad de México, con el 13,5 y 7,9\% del consumo nacional. Otras entidades importantes en el consumo fueron Veracruz $(6,8 \%)$, Jalisco (6,5 \%), Puebla $(5,1 \%)$ y Nuevo León $(4,1 \%)$. El comercio exterior de la naranja en fresco no es considerable en México, ya que las importaciones y exportaciones en 2017 se ubicaron en 27 y 55 mil t, respectivamente (Sistema de Información Agroalimentaria y Pesquera, 2018).

Igual que todos los productos del sector agrícola (Food and Agriculture Organization, 2010), la naranja se caracteriza por presentar una fuerte volatilidad en los precios en todos sus niveles (productor, mayoreo y consumidor). En el periodo 1980-2016 se presentó una relación negativa entre el precio al mayoreo en la central de abasto de la Ciudad de México y la producción nacional de naranja. Con un análisis de regresión, en donde la producción (en miles de t) fue la variable independiente y el precio la variable dependiente, se obtuvo un $\mathrm{R}^{2}$ de 0,62 , un intercepto de 14618 y un coeficiente de -2,18. Estos resultados señalan que si la producción de naranja aumenta en $1000 \mathrm{t}$, su precio disminuye en 2,18 pesos por tonelada $\left(\$ \mathrm{t}^{-1}\right)$, equivalente a $0,14 \mathrm{USD} \$ \mathrm{t}^{-1}$.

La volatilidad de precios es nociva para productores y consumidores. La caída de los precios es negativa para los productores porque ante excesos de producción deben vender por debajo de sus costos incurriendo en pérdidas. En el periodo 2014-2016, datos promedio sobre precios y rendimientos reportados por el Servicio de Información 
Agroalimentaria y Pesquera (SIAP) indicaron que el ingreso en las zonas de riego y temporal fue de 2500 y 1119 USD $\$ h^{-1}$, respectivamente. Los costos de producción dependen de las condiciones de producción de la localidad, así como de prácticas agrícolas implementadas en las huertas tales como riego, control de plagas y otras, es por

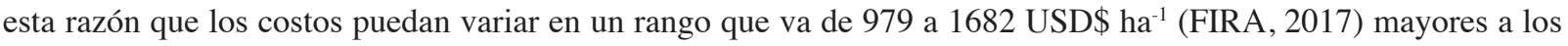
ingresos que se obtienen en las zonas de temporal.

Los datos anteriores señalan que, en algunas huertas de temporal, los ingresos obtenidos por la venta de la naranja son menores a los costos, lo cual indica que los productores incurren en pérdidas. La disminución del ingreso del productor no es recomendable por los efectos negativos sobre la pobreza. Para los consumidores la volatilidad de precios es perjudicial en periodos de precios altos porque incurren en un gasto mayor. Los precios altos se observan cuando el producto en el mercado es escaso.

El precio en un mercado que funciona bajo competencia perfecta, como es el caso de la naranja, depende del comportamiento de la oferta y la demanda (Varian, 1992). Los precios de la naranja en México son volátiles porque la demanda de naranja es inelástica, la elasticidad precio de la demanda de la naranja es de -0,24 para el consumo en fresco y de -0,35 para la demanda industrial (Bautista-Mayorga, 2017). Cambios pequeños en la oferta de naranja determinan una fuerte variabilidad en los precios, debido a la inelasticidad de la curva de demanda. El desplazamiento de la curva de demanda también afecta el precio de la naranja; por ejemplo, el aumento en el ingreso del cosumidor, en la población y en el precio de bienes sustitutos, como la toronja (Citrus paradisi Macf.) y el mango (Mangifera indica L.), incrementan la demanda a cualquier nivel de precio.

El Comité Sistema-Producto de Cítricos del Estado de Veracruz reportó que la agroindustria de la naranja en México inició sus actividades en 1972 con la instalación de una planta productora de jugos en Nuevo León; en 1973 se ubicó la segunda y, desde entonces, dicha industria empezó a crecer de tal forma que en 1980 existían nueve plantas, en 1991 veintidós, y en 1994, existían veintiocho plantas de jugo en México (Comité Sistema Productor Cítricos del Estado de Veracruz, 2009). En el periodo 2014-2016 la industria demandó en promedio 1617 Mt de naranja, lo que representó más del $30 \%$ del consumo nacional.

Con relación al problema de los precios, sería recomendable la participación de la agroindustria citrícola, quien utiliza la naranja fresca para producir jugo. El mercado de productos agrícolas procesados se caracteriza por la concentración del poder de compra (monopsonio u oligopsonio), en algunas regiones; se trata de productos procesados antes de llegar al consumidor final (Food and Agriculture Organization, 2004). Una parte del consumo de naranja se encuentra entre los productos que deben ser procesados para llegar al consumidor.

Debido a que el almacenamiento de la naranja en fresco como medida de control de la oferta no es posible por ser un producto perecedero, entonces la agroindustria podría adquirir los excesos de oferta temporales de naranja para su industrialización a un precio que garantice un margen de ganancia al productor. La agroindustria actuaría de emergencia cuando el mercado no pueda absorber los excesos de naranja. La situación anterior es posible si se considera que a la larga los productores y procesadores necesitan mantener relaciones mutuas. Un precio bajo de la naranja estimularía a los productores a cambiar hacia otros productos en el largo plazo. Los productores suelen quejarse de que los industriales pagan precios bajos o que rehúsan trasladarles beneficios proporcionales a los aumentos en los precios del producto procesado (Food and Agriculture Organization, 2004).

En relación con la agroindustria, la Organización de Naciones Unidas para la Alimentación y la Agricultura (Food and Agriculture Organization, 2004) propone las siguientes medidas como mecanismo de control de los precios: a) Procesamiento del producto por los productores a través de empresas de su propiedad que pueden ofrecer mejores precios por la materia prima; b) Legislación antimonopólica, debe disponerse de soluciones legales para los casos de abuso del poder por parte de monopsonios y oligopsonios y; 3) Acuerdos sobre precios avalados por el gobierno, en los cuales este gestione los precios entre los agentes.

La relación negativa entre el precio y la cantidad señala que el comportamiento de la producción también impacta los precios. La producción de naranja es igual a la superficie por el rendimiento y, por lo tanto, el 
comportamiento de estas dos variables impacta a los precios. El comportamiento del rendimiento de la naranja está asociado al riesgo de la empresa, determinado por las condiciones climáticas y fitosanitarias. Las inundaciones, las sequías, las granizadas y la presencia de plagas y enfermedades inciden sobre la magnitud del rendimiento. En cambio, la superficie depende de factores económicos como el precio de la naranja, el precio de los cultivos que compiten por el uso de los recursos y el precio de los insumos usados en la producción.

El comportamiento de la producción depende de la superficie, variable que sí se puede planear en el largo plazo, y de esta manera se puede incidir sobre la producción. En el periodo 1992-2016, la superficie cosechada de naranja fue de 330 mil ha, con superficies mínima y máxima de 272 y 349 mil ha. La diferencia entre la superficie promedio y máxima fue mayor a 18 mil ha, lo cual indica la fuerte variación que se presenta en esta variable.

Para evitar la volatilidad en los precios, el comportamiento de la superficie sembrada de naranja no debería fluctuar a través de los años, debería ser uniforme en el tiempo. La fuerte concentración de la producción a nivel estatal, en donde el estado de Veracruz produce más del $50 \%$ de la producción, supone que sí es posible una planeación adecuada en la superficie. La Secretaría de Agricultura, Ganadería, Desarrollo Rural, Pesca y Alimentación (SAGARPA) y el sistema producto de la naranja a nivel nacional, deben instrumentar las medidas necesarias para lograr planear la superficie sembrada de naranja, además de crear conciencia en los productores sobre los mayores ingresos que se lograrían si se evitan los excesos de oferta de la fruta. Una vez observada la importancia del problema anterior, el objetivo de este trabajo fue determinar cómo la demanda de la industria y la superficie sembrada de naranja afectan los precios al productor, al consumidor y al mayoreo.

\section{Materiales y métodos}

\section{El modelo}

Para determinar cómo la demanda de la industria y la superficie sembrada de naranja afectan los precios al productor, al consumidor y al mayoreo, se formuló un modelo sintético del mercado de la naranja en México, el cual usa parámetros existentes para crear una estructura que replica el valor de las variables endógenas, dados los valores de elasticidades predeterminadas y variables exógenas (Rude et al., 2007). Los parámetros del modelo sintético no son estimados en el mismo modelo, se obtienen de la literatura o son estimados econométricamente, pero de forma separada del modelo, y se pueden construir en hojas de cálculo (Francois y Reinert, 1997). El modelo usa información anual y nacional, y para mayor representatividad, los indicadores nacionales usados, en todos los casos, se refieren al promedio de tres años. Cualquier valor promedio se obtiene de datos anuales y nacionales observados en 2014, 2015 y 2016.

Las razones que justifican la selección de un modelo sintético son: 1) permite captar la relación entre las variables del estudio: producción, superficie, demanda (en fresco e industrial), y precios (productor, consumidor y mayoreo); 2) las variables más importantes como los precios son independientes en algunas relaciones y dependientes en otras; 3) permite diferenciar las variables endógenas de las exógenas; 4) permite realizar escenarios a través de cambios en las variables exógenas que impactan a las variables endógenas; 5) su formulación en hojas de cálculo facilita la elaboración de escenarios (García-Salazar y Ramírez-Jaspeado, 2013). El modelo permite medir los efectos sobre los precios de cambios en la superficie sembrada, demanda industrial y el cambio en algún factor que afecta la oferta o la demanda.

La construcción del modelo sintético del mercado de la naranja se basó en la teoría microeconómica. El mercado de la naranja está integrado por la producción en riego y temporal, el consumo en fresco e industrial, los precios al productor, mayoreo y consumidor, y las exportaciones e importaciones. Basados en la teoría microeconómica y en evidencia empírica (García et al., 2003; Tomek y Robinson, 2003) la producción en riego y temporal y la demanda en fresco e industrial se pueden representar de la siguiente manera: 


$$
\begin{aligned}
& Q P N R={ }_{\alpha, 0}+{ }_{\alpha 1} P P N R+{ }_{\alpha 2} P P C R+{ }_{\alpha 3} S M R+{ }_{\alpha 4} D A R+{ }_{\alpha 5} \text { TEM } \\
& Q P N T={ }_{\gamma^{\prime} 0}+{ }_{\gamma 1} P P N T+{ }_{\gamma 2} P P C T+{ }_{\gamma 3} S M R+{ }_{\gamma 4} P P+{ }_{\gamma 5} T E M \\
& Q D N F={ }_{\beta^{\prime} 0}+{ }_{\beta 1} P C N+{ }_{\beta 2} I N G P+{ }_{\beta 3} P C L+{ }_{\beta 4} P C T+{ }_{\beta 5} P C M \\
& Q D N I={ }_{\delta^{\prime} 0}+{ }_{\delta 1} P C J N+{ }_{\delta 2} I N G R+{ }_{\delta 3} P C J U+{ }_{\delta 4} P R E F
\end{aligned}
$$

Donde: QPNR es la cantidad producida de naranja bajo riego, $t$; PPNR es el precio promedio al productor de naranja bajo riego, $\$ \mathrm{t}^{-1}$; PPCR es el precio promedio al productor de café cereza bajo riego, $\$ \mathrm{t}^{-1}$; SMR es el salario mínimo promedio, $\$$ día $^{-1}$; DAR es la disponibilidad de agua para riego, miles $\mathrm{m}^{-3}$; TEM es la temperatura promedio anual, ${ }^{\circ} \mathrm{C}$; QPNT es la cantidad producida de naranja bajo temporal, t; PPNT es el precio promedio al productor de naranja bajo temporal, $\$ \mathrm{t}^{-1}$; PPCT es el precio promedio al productor de café cereza bajo temporal, $\$ \mathrm{t}^{-1}$; PP es la precipitación promedio anual, mm; QDNF es la cantidad demandada de naranja en fresco, t; PCN es el precio promedio al consumidor de naranja, $\$ \mathrm{t}^{-1}$; INGP es el ingreso per cápita, millones de \$; PCL, PCT y PCM son los precios promedio al consumidor de limón (Citrus latifolia tanaka), toronja y mango, $\$ \mathrm{t}^{-1}$; QDNI es la cantidad demandada de naranja para la industria, $t$; PCJN es el precio al consumidor de jugo de naranja, $\$ \mathrm{t}^{-1}$; PCJU es el precio al consumidor de jugo de uva, $\$ \mathrm{t}^{-1} \mathrm{y}$; PREF es el precio al consumidor de refresco de sabor, $\$ \mathrm{t}^{-1}$. Todas las variables monetarias (expresadas en $\$$ ) fueron deflactadas por el índice nacional de precios al consumidor (INPC) y el índice nacional de precios al productor (INPP).

$$
\text { Suponiendo que }{ }_{\alpha 0}={ }_{\alpha^{\prime} 0}+{ }_{\alpha 2} P P C+{ }_{\alpha 3} S M R+{ }_{\alpha 4} D A R+{ }_{\alpha 5} T E M P
$$

Y haciendo el mismo supuesto para las tres ecuaciones restantes, el sistema de ecuaciones 1-4 se transforma en:

$$
\begin{aligned}
& Q P N R={ }_{\alpha 0}+{ }_{\alpha l} P P N R \\
& Q P N T={ }_{\gamma 0}+{ }_{\gamma l} P P N T \\
& \text { QDNF }={ }_{\beta 0}+{ }_{\beta I} P C N \\
& \text { QDNI }={ }_{\delta 0}+{ }_{\delta I} P C J N
\end{aligned}
$$

La relaciones entre los niveles de precios se pueden expresar de la siguiente manera:

$$
\begin{aligned}
& \text { PPNR }={ }_{\theta 0}+{ }_{\theta 1} P M A Y N \\
& P P N T={ }_{\varepsilon 0}+{ }_{\varepsilon I} P M A Y N \\
& P C N={ }_{\lambda 0}+{ }_{\lambda l} P M A Y N \\
& P C J N={ }_{\eta 0}+{ }_{\eta I} P M A Y N
\end{aligned}
$$

Donde, PMAYN es el precio al mayoreo de la naranja, en $\$ \mathrm{t}^{-1}$.

En las centrales de abasto es donde los mayoristas (agentes que llevan a cabo el transporte y demás prácticas de comercialización de naranja) conectan a los productores (ubicados en las zonas agrícolas) con los consumidores finales (localizados en las ciudades); por lo tanto, los precios al productor y al consumidor (ecuaciones 10-13) dependen del precio al mayoreo (Calderón et al., 2004).

El equilibrio en el mercado se obtiene igualando la disponibilidad de naranja [producción más importaciones de naranja $(\mathrm{QMN})$ ] con la demanda total [suma de la demanda en fresco, demanda industrial, y mermas de naranja (QMEN) y exportaciones $(\mathrm{QXN})]$, esto es:

$$
Q P N R+Q P N T+Q M N=Q D N F+Q D N I+Q M E N+Q X N
$$


$\mathrm{Al}$ sustituir las ecuaciones 6-9 en $14 \mathrm{y}$, posteriormente, las ecuaciones 10-13, se puede determinar el precio al mayoreo con base en los factores que afectan a la oferta y a la demanda; en este caso la superficie y la demanda industrial son variables endógenas determinadas por los factores que afectan la oferta y la demanda; es decir, no son influenciadas por una acción gubernamental o por la organización de los productores.

$$
\text { PMAYN }=\frac{(\alpha 0+\alpha 1 \theta 0+\gamma 0+\gamma 1 \varepsilon 0-\beta 0-\beta 1 \lambda 0-\delta 0-\delta 1 \eta 0-Q M E N+Q M N-Q X N)}{\beta 1 \lambda 1+\delta 1 \eta 1-\alpha 1 \theta 1-\gamma 1 \varepsilon 1}
$$

La ecuación 15 indica que el precio al mayoreo de la naranja depende de los factores que afectan a la producción y a la demanda. Un aumento en las exportaciones significa una demanda y un precio mayor. Un aumento en las importaciones aumentará la disponibilidad, bajando el precio.

Para lograr que la superficie sea una variable exógena, la producción de naranja (ecuaciones 1 y 2 o 6 y 7 ) se puede expresar considerando que esta variable es igual a la superficie por el rendimiento:

$$
\left.S N R * R N R+S N T * R N T+Q M N=\left[_{\beta 0}+{ }_{\beta 1}\left({ }_{\lambda 0}+{ }_{\lambda l} P M A Y N\right)\right]+\left[_{\delta 0}+{ }_{\delta 1}\left({ }_{\eta 0}+{ }_{\eta I} P M A Y N\right)\right]+Q M E N+Q X N \quad 16\right)
$$

Donde, SNR y SNT es la superficie de naranja en riego y temporal, en hectáreas y; RNR y RNT es el rendimiento de la naranja en riego y temporal, $\mathrm{t} \mathrm{ha}^{-1}$. El precio que mantiene en equilibrio el mercado se obtiene despejando PMAYN:

$$
\text { PMAYN }=\frac{(S N R * R N R+S N T * R N T-\beta 0-\beta 1 \lambda 0-\delta 0-\delta 1 \eta 0-Q M E N+Q M N-Q X N)}{\beta 1 \lambda 1+\delta 1 \eta 1}
$$

La ecuación 17 establece una relación negativa entre el precio al mayoreo y la superficie sembrada de naranja. Un aumento en la superficie bajará el precio y viceversa. También establece una relación negativa entre el rendimiento y el precio al mayoreo, mejoras en el rendimiento por hectáreas bajarán el precio al mayoreo por la mayor disponibilidad de producto.

Suponiendo que la superficie sembrada y la demanda industrial son fijas, el precio al mayoreo queda expresado de la siguiente manera:

$$
\mathrm{PMAYN}=\frac{(S N R * R N R+S N T * R N T-\beta 0-\beta 1 \lambda 0-Q D N I-Q M E N+Q M N-Q X N)}{\beta 1 \lambda 1}
$$

Si la producción es considerada como variable endógena y depende de los factores que afectan a la oferta, entonces el precio al mayoreo de la naranja queda expresado como:

$$
\text { PMAYN }=\frac{(\alpha 0+\alpha 1 \theta 0+\gamma 0+\gamma 1 \varepsilon 0-\beta 0-\beta 1 \lambda 0-Q D N I-Q M E N+Q M N-Q X N)}{\beta 1 \lambda 1-\alpha 1 \theta 1-\gamma 1 \varepsilon 1}
$$

La ecuación 19 establece una relación positiva entre el precio al mayoreo de la naranja y la demanda por parte de la industria. Un aumento en la demanda industrial elevará el precio al mayoreo y viceversa.

Asumiendo que $\mathrm{Y}$ y $\mathrm{X}$ son vectores que contienen a las variables endógenas y exógenas; y que A y B son matrices de parámetros asociados a las variables endógenas y exógenas, las ecuaciones 6-13 y la ecuación 15 (o las ecuaciones 17,18 y 19$)$ se puede expresar en forma matricial:

$A Y=B X$

El vector Y contiene las variables endógenas: producción en riego, producción en temporal, demanda para consumo en fresco, demanda industrial, precio al productor en riego, precio al productor en temporal, precio 
al consumidor de la naranja en fresco, precio al consumidor de jugo naranja y precio al mayoreo de naranja. El vector X contiene: el intercepto de las funciones de la oferta (riego y temporal), el intercepto de las funciones de la demanda (en fresco e industrial), el intercepto de las ecuaciones de transmisiones del precio, las exportaciones y las importaciones de naranja. El término "transmisión de precios" hace referencia a la forma y velocidad de ajuste de los precios domésticos ante cambios en los precios internacionales (Jaramillo-Villanueva \& Benítez-García, 2016).

La solución del sistema se obtiene despejando el vector Y, esto es:

$\mathrm{Y}=A^{-1} B X$

En donde $\mathrm{A}^{-1}$ es la matriz inversa de la matriz $\mathrm{A}$.

Al usar el valor observado de las variables exógenas en el año de estudio, el modelo permite replicar el valor de las variables endógenas. Una vez validado el modelo, que consiste en comparar los valores observados de las variables endógenas con los valores estimados por el mismo, se realizaron los siguientes escenarios: 1) La demanda de la industria aumenta en $5 \%$; 2) La demanda de la industria aumenta en $10 \%$; 3) La superficie en riego y temporal disminuye en $5 \%$ y; 4) La superficie en riego y temporal disminuye en $10 \%$. Los escenarios 1 y 2 consideran los siguientes dos supuestos: a) Cuando la producción es fija (igual a la superficie por el rendimiento) y; b) Cuando la producción es variable y depende de los factores que afectan a la oferta. Los escenarios 3 y 4 consideran a la demanda industrial de naranja como una variable endógena. Para expresar los resultados en dólares americanos, el valor de las variables monetarias fue dividido por el tipo de cambio (\$ por USD\$) promedio en el periodo 2014 a 2016.

Para obtener las ecuaciones de la oferta, de la demanda y de la transmisión de precios se usaron las elasticidades precio de la oferta y de la demanda de naranja, las elasticidades de ingreso, las elasticidades cruzadas y las elasticidades de transmisión de precios, las cantidades ofertadas y demandadas y los precios al productor, al mayoreo y al consumidor.

\section{Datos}

Las elasticidades provinieron de Bautista-Mayorga (2017). La información de superficie en riego y temporal, rendimiento en riego y temporal, consumo en fresco y precios promedios al productor en los años 2014, 2015 y 2016 se obtuvieron del Sistema de Información Agroalimentaria y Pesquero (2017). La producción se estimó usando la superficie y el rendimiento. Los datos del salario mínimo provinieron de la Comisión Nacional de Salarios Mínimos (Comisión Nacional de Salarios Mínimos [CONASAMI], 2017) y el ingreso per cápita se obtuvo del Instituto Nacional de Estadística y Geografía (Instituto Nacional de Estadística y Geografía, 2017a).

La precipitación pluvial promedio anual (que incluye solo a estados donde se produce naranja) se obtuvo de la Secretaría del Medio Ambiente y Recursos Naturales (2017); la temperatura promedio anual se obtuvo de la Comisión Nacional del Agua (Comisión Nacional del Agua, 2017). El precio al consumidor de la naranja, limón, toronja y mango se obtuvieron de la Procuraduría Federal del Consumidor. Los precios al consumidor del jugo de naranja, uva y refresco se obtuvieron del Instituto Nacional de Estadística y Geografía (2017b), y los precios al mayoreo (que incluyen el precio promedio en las centrales de abasto de la Ciudad de México, Guadalajara y Monterrey) se obtuvieron del Sistema Nacional de Información e Integración de Mercados (2017). El Índice Nacional de Precios al Consumidor y el Índice Nacional de Precios al Productor fueron obtenidos del Instituto Nacional de Estadística y Geografía (2017c).

Agron. Mesoam. 32(1):209-223, enero-abril, 2021 ISSN 2215-3608 doi:10.15517/am.v32i1.40679 


\section{Resultados}

Debido a que cada coeficiente del modelo se estimó con base en las elasticidades, los precios y las cantidades observadas de naranja, cada uno de los coeficientes presenta el signo esperado de acuerdo con la teoría económica. El valor de los coeficientes de las ecuaciones, de la oferta, de la demanda y de la transmisión de precios se puede observar en el Cuadro 1.

Cuadro 1. Coeficientes estimados del modelo sintético del mercado de la naranja (Citrus sinensis L.) en México, $2014-2016$.

Table 1. Estimated coefficients of the synthetic model of the orange (Citrus sinensis L.) market in Mexico, 2014-2016.

\begin{tabular}{|c|c|c|c|c|c|c|}
\hline \multirow{2}{*}{$\begin{array}{l}\text { Variable endógena } \\
\text { QPNR }\end{array}$} & \multicolumn{6}{|c|}{ Variables exógenas } \\
\hline & a'0 & PPNR & PPCR & SMR & DAR & TEM \\
\hline & -1164862 & 44,39651 & $-5,52752$ & $-5605,82$ & 0,008349 & 126717,4 \\
\hline \multirow[t]{2}{*}{ QPNT } & $\gamma^{\prime} 0$ & PPNT & PPCT & SMR & $\mathrm{PP}$ & TEM \\
\hline & 384802 & 161,2676 & $-29,6857$ & -19827 & 150,556 & 176839,1 \\
\hline \multirow[t]{2}{*}{ QDNF } & $\beta ’ 0$ & PCN & INGP & PCL & PCT & PCM \\
\hline & 2578521 & $-56,4965$ & 7,360311 & $-8,26741$ & $-46,1618$ & 17,68277 \\
\hline \multirow[t]{2}{*}{ QDNI } & $\delta^{\prime} 0$ & PCJN & INGP & PCJU & PREF & \\
\hline & 1147833 & $-43,8841$ & 8,119772 & 32,54034 & $-105,452$ & \\
\hline \multirow[t]{2}{*}{ PPNR } & $\phi 0$ & PMAYN & & & & \\
\hline & 1405 & 0,11 & & & & \\
\hline \multirow[t]{2}{*}{ PPNT } & $\varepsilon 0$ & PMAYN & & & & \\
\hline & -180 & 0,23 & & & & \\
\hline \multirow[t]{2}{*}{ PCN } & $\lambda 0$ & PMAYN & & & & \\
\hline & 6782 & 0,66 & & & & \\
\hline \multirow[t]{2}{*}{ PCJN } & $\eta 0$ & PMAYN & & & & \\
\hline & 7426 & 0,8 & & & & \\
\hline
\end{tabular}

QPNR: cantidad producida de naranja bajo riego, a'0: intercepto de la cantidad producida de naranja bajo riego, PPNR: precio promedio al productor de naranja bajo riego, PPCR: precio promedio al productor de café cereza bajo riego, SMR: salario mínimo promedio, DAR: disponibilidad de agua para riego, TEM: temperatura promedio anual, QPNT: cantidad producida de naranja bajo temporal, $\gamma$ '0: intercepto de la cantidad producida de naranja bajo temporal, PPNT: precio promedio al productor de naranja bajo temporal, PPCT: precio promedio al productor de café cereza bajo temporal, PP: precipitación promedio anual, QDNF: cantidad demandada de naranja en fresco, $\beta$ '0: intercepto de la cantidad demandada de naranja en fresco, PCN: precio promedio al consumidor de naranja, INGP: ingreso per cápita, PCL: precio promedio al consumidor de limón, PCT: es el precio promedio al consumidor de toronja, PCM: precio promedio al consumidor de mango, QDNI: cantidad demandada de naranja para la industria, $\delta$ ' 0 : intercepto de la cantidad demanda de naranja para la industria, PCJN: precio al consumidor de jugo de naranja, PCJU: precio al consumidor de jugo de uva, PREF: precio al consumidor de refresco de sabor, $\phi 0$ : intercepto del precio promedio al productor de naranja bajo riego, PMAYN: precio al mayoreo de la naranja, $\varepsilon 0$ : intercepto del precio promedio al productor de naranja bajo temporal, $\lambda 0$ : intercepto del precio promedio al consumidor de naranja, $\eta 0$ : intercepto del precio promedio al consumidor de jugo de naranja / QPNR: quantity of orange produced under irrigation, a'0: intercept of the quantity of orange produced under irrigation, PPNR: average price to the producer of orange under irrigation, PPCR: average price to the producer of low cherry coffee under irrigation, SMR: average minimum wage, DAR: availability of water for irrigation, TEM: annual average temperature, QPNT: produced quantity of temporary low orange, $\gamma^{\prime} 0$ : intercept of the quantity produced of orange temporary low, PPNT: average price to the producer of temporary low orange, PPCT: average price to the producer of temporary low cherry coffee, PP: average annual rainfall, QDNF: quantity demanded of fresh orange, $\beta$ ' 0 : intercept of the quantity demanded of fresh orange, PCN: average consumer price of orange, INGP: per capita income, PCL: average consumer price of lemon, PCT: average price at Grapefruit consumer, PCM: Average consumer price of mango, QDNI: quantity demanded of orange for the industry, $\delta$ '0: intercept of the quantity demanded of orange for the industry, PCJN: orange juice consumer price, PCJU: consumer price of grape juice, PREF: consumer price of flavor soda, $\phi 0$ : intercept of the average price to the orange producer under irrigation, PMAYN: wholesale price of the orange, $\varepsilon 0$ : intercept of the average price to the temporary low orange producer, $\lambda 0$ : intercept of the average consumer price of orange, $\eta 0$ : intercept of the average consumer price of orange juice. 
Una vez que el modelo sintético en su forma matricial se ha construido de forma correcta, permite replicar con exactitud el valor observado de las variables endógenas. En este caso, el modelo permitió replicar el valor observado de la producción en riego y temporal, el consumo en fresco, el consumo industrial y los diferentes niveles de precios (productor, mayoreo y consumidor).

El valor estimado de las variables endógenas es igual al valor observado. En el año promedio 2014-2016 la producción en riego y temporal de naranja fue de 1596 y 3207 miles de t, respectivamente. El consumo de naranja en fresco fue de 2658 mil t y la industria realizó un consumo de 1617 mil t. En el año seleccionado las mermas de naranja en el proceso de comercialización fueron 499 mil t (Cuadro 2).

Cuadro 2. Efectos de un aumento en la demanda industrial sobre el mercado de naranja (Citrus sinensis L.) en México, $2014-2016$. Table 2. Effect of an increase in industrial demand on the orange (Citrus sinensis L.) market in Mexico, 2014-2016.

\begin{tabular}{|c|c|c|c|c|c|c|c|}
\hline \multirow{4}{*}{ Variable endógena } & \multirow{3}{*}{$\begin{array}{c}\text { Situación } \\
\text { base }\end{array}$} & \multicolumn{2}{|c|}{$\begin{array}{l}\text { Aumento en \% en la } \\
\text { demanda industrial: }\end{array}$} & \multicolumn{4}{|c|}{ Cambio con respecto a la situación base } \\
\hline & & 5 & 10 & 5 & 10 & 5 & 10 \\
\hline & & \multicolumn{4}{|c|}{ Miles de $\mathrm{t}$ y USD\$ $\mathrm{t}^{-1}$} & \multicolumn{2}{|c|}{$\%$} \\
\hline & \multicolumn{7}{|c|}{ Supuesto a: La producción es constante } \\
\hline QPNR & 1596 & 1596 & 1596 & 0 & 0 & 0 & 0 \\
\hline QPNT & 3207 & 3207 & 3207 & 0 & 0 & 0 & 0 \\
\hline QMEN & 499 & 499 & 499 & 0 & 0 & 0 & 0 \\
\hline QDNF & 2658 & 2577 & 2497 & -81 & -162 & -3 & $-6,1$ \\
\hline PPNR & 135 & 150 & 165 & 15 & 30 & 11,1 & 22,1 \\
\hline PPNT & 87 & 118 & 150 & 31 & 62 & 35,8 & 71,6 \\
\hline $\mathrm{PCN}$ & 707 & 797 & 887 & 90 & 179 & 12,7 & 25,3 \\
\hline PCJN & 808 & 916 & 1025 & 109 & 217 & 13,5 & 26,9 \\
\hline \multirow[t]{2}{*}{ PMAYN } & 428 & 564 & 700 & 136 & 272 & 31,7 & 63,4 \\
\hline & \multicolumn{7}{|c|}{ Supuesto b: La producción es variable } \\
\hline QPNR & 1596 & 1601 & 1606 & 5 & 11 & 0,3 & 0,7 \\
\hline QPNT & 3207 & 3247 & 3287 & 40 & 80 & 1,2 & 2,5 \\
\hline QMEN & 499 & 504 & 509 & 5 & 9 & 0,9 & 1,9 \\
\hline QDNF & 2658 & 2618 & 2578 & -40 & -80 & $-1,5$ & -3 \\
\hline PPNR & 135 & 143 & 150 & 7 & 15 & 5,5 & 11 \\
\hline PPNT & 87 & 103 & 118 & 16 & 31 & 17,8 & 35,7 \\
\hline $\mathrm{PCN}$ & 707 & 752 & 797 & 45 & 89 & 6,3 & 12,6 \\
\hline PCJN & 808 & 862 & 916 & 54 & 108 & 6,7 & 13,4 \\
\hline PMAYN & 428 & 496 & 563 & 68 & 135 & 15,8 & 31,6 \\
\hline
\end{tabular}

Las cantidades (QPNR, QPNT, QMEN y QDNF) y los precios (PPNR, PPNT, PCN, PCJN y PMAYN) en las columnas 2-6 están expresadas en miles de $\mathrm{t}$ y en USD $\mathrm{t}^{-1}$, respectivamente. Los valores de las columnas 7 y 8 están expresados en porcientos (\%).

QPNR: cantidad producida de naranja bajo riego, a'0: intercepto de la cantidad producida de naranja bajo riego, QPNT: cantidad producida de naranja bajo temporal, QDNI: cantidad demandada de naranja para la industria, QDNF: cantidad demandada de naranja en fresco, PPNR: precio promedio al productor de naranja bajo riego, PPNT: precio promedio al productor de naranja bajo temporal, PCN: precio promedio al consumidor de naranja, PCJN: precio al consumidor de jugo de naranja, PMAYN: precio al mayoreo de la naranja / QPNR: quantity produced of orange under irrigation, a'0: intercept of the quantity produced of orange under irrigation, QPNT: quantity produced of orange under temporary, QDNI: quantity demanded of orange for the industry, QDNF: the quantity demanded of fresh orange, PPNR: average price to the producer of orange under irrigation, PPNT: average price to the producer of temporary low orange, PCN: average price to the consumer of orange, PCJN: consumer price of orange juice, PMAYN: wholesale price of orange. 
Los resultados de los escenarios 3 y 4 se presentan en el Cuadro 3. Los datos reportados por el Servicio de Información Agroalimentaria y Pesquera (2017) indican que el precio de la naranja en las zonas de riego y temporal fue de 135 y 87 USD $\$ t^{-1}$, respectivamente. La diferencia en los precios se debe a la mejor calidad de la naranja obtenida en las zonas de riego. Una vez que la naranja se compra y obtiene en las zonas de producción, esta se lleva a las centrales de abasto de las ciudades para ser vendida al mayoreo, en el año de análisis el precio al mayoreo de la naranja fue de $428 \mathrm{USD} \$ \mathrm{t}^{-1}$. El consumidor final paga el precio mayor en la cadena productiva. Se pudo observar que el precio al consumidor de la naranja en fresco fue de 707 USD $\$ t^{-1}$; en tanto el precio del jugo de naranja fue

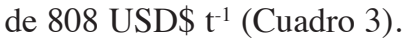

Cuadro 3. Efectos de una disminución en la superficie sobre el mercado de naranja (Citrus sinensis L.) en México, 2014-2016. Table 3. Effect of a decrease in the surface on the orange (Citrus sinensis L.) market in Mexico, 2014-2016.

\begin{tabular}{|c|c|c|c|c|c|c|c|}
\hline \multirow{3}{*}{ Variable endógena } & \multirow{3}{*}{$\begin{array}{l}\text { Situación } \\
\text { base }\end{array}$} & \multicolumn{2}{|c|}{$\begin{array}{c}\text { Disminución en \% en la } \\
\text { superficie: }\end{array}$} & \multicolumn{4}{|c|}{ Cambio respecto a la situación base } \\
\hline & & 5 & 10 & 5 & 10 & 5 & 10 \\
\hline & & \multicolumn{4}{|c|}{ Miles de t y USD\$ t-1 } & \multicolumn{2}{|l|}{$\%$} \\
\hline QPNR & 1596 & 1516 & 1436 & -80 & -160 & $-5,0$ & $-10,0$ \\
\hline QPNT & 3207 & 3046 & 2886 & -160 & -321 & $-5,0$ & $-10,0$ \\
\hline QMEN & 499 & 474 & 450 & -25 & -50 & $-5,0$ & $-10,0$ \\
\hline QDNF & 2658 & 2547 & 2437 & -111 & -222 & $-4,2$ & $-8,3$ \\
\hline QDNI & 1617 & 1512 & 1408 & -104 & -209 & $-6,5$ & $-12,9$ \\
\hline PPNR & 135 & 156 & 176 & 20 & 41 & 15,2 & 30,3 \\
\hline PPNT & 87 & 130 & 173 & 43 & 86 & 49,1 & 98,2 \\
\hline PCN & 707 & 830 & 953 & 123 & 246 & 17,4 & 34,7 \\
\hline PCJN & 808 & 957 & 1106 & 149 & 298 & 18,4 & 36,9 \\
\hline PMAYN & 428 & 614 & 801 & 186 & 372 & 43,5 & 87,0 \\
\hline
\end{tabular}

Las cantidades (QPNR, QPNT, QMEN, QDNF y QDNI) y los precios (PPNR, PPNT, PCN, PCJN y PMAYN) en las columnas 2-6 están expresadas en miles de $\mathrm{t}$ y en USD $\$ \mathrm{t}^{-1}$, respectivamente. Los valores de las columnas 7 y 8 están expresados en porcientos (\%). QPNR: es la cantidad producida de naranja bajo riego, a'0: intercepto de la cantidad producida de naranja bajo riego, QPNT: es la cantidad producida de naranja bajo temporal, QDNI: es la cantidad demandada de naranja para la industria, QDNF: es la cantidad demandada de naranja en fresco, PPNR: es el precio promedio al productor de naranja bajo riego, PPNT: es el precio promedio al productor de naranja bajo temporal, PCN: es el precio promedio al consumidor de naranja, PCJN: es el precio al consumidor de jugo de naranja, PMAYN: es el precio al mayoreo de la naranja / QPNR: is the quantity produced of orange under irrigation, a'0: intercept of the quantity produced of orange under irrigation, QPNT: is the quantity produced of orange under temporary, QDNI: is the quantity demanded of orange for the industry, QDNF: the quantity demanded of fresh orange, PPNR: is the average price to the producer of orange under irrigation, PPNT: is the average price to the producer of temporary low orange, PCN: is the average price to the consumer of orange, PCJN: is the consumer price of orange juice, PMAYN: is the wholesale price of orange.

\section{Discusión}

Un aumento de $5 \%$ en la demanda de naranja que se usa como materia prima en la industria de jugo, sería un factor que aumentaría el precio al productor. La demanda industrial de naranja pasaría de 1617 a 1698 miles de t, lo cual representaría un aumento de 81000 t que tendría que procesar la industria. Si se mantienen constantes la superficie y el rendimiento de naranja, un aumento de $5 \%$ en la demanda industrial ocasionaría que el precio al 
mayoreo pase de 428 a 564 USD $\$ t^{-1}$, lo que significaría un crecimiento de $31,7 \%$. Los cambios en el precio al mayoreo se transmitirían a nivel del productor, los cuales crecerían en $11,1 \%$ en las zonas de riego y 35,8 \% en las zonas de temporal (Cuadro 2). El cambio en el precio al mayoreo de la naranja en las centrales de abasto también se transmitiría a nivel del consumidor, ya que el precio que tendría que pagar este agente pasaría de 707 a 797 USD $\mathrm{t}^{-1}$. Por el cambio en los precios, la demanda de naranja en fresco disminuiría en 81000 t.

Los resultados obtenidos en esta investigación fueron similares a los encontrados por González-Rojas et al. (2011) para el mercado mundial de maíz (Zea mays L.); los autores señalaron que un aumento en la demanda mundial de maíz para producir etanol, por efecto de un aumento en el precio del petróleo, incrementaría los precios del grano en Estados Unidos y el resto del mundo.

Si se considera a la producción como una función de la oferta (ecuaciones 1 y 5), los efectos de un aumento en la demanda industrial serían más moderados. El aumento de la demanda industrial de naranja en $5 \%$ aumentaría el precio al mayoreo en 15,8 \%, notoriamente inferior a la situación en donde la superficie y la producción se mantienen constantes. Los precios al productor aumentarían en 5,5\% en las zonas riego y en 17,8 \% en las zonas de temporal, en tanto que los precios al consumidor de la naranja en fresco y el jugo aumentaría en 6,3 y 6,7 \%, en relación con la situación base. La razón económica que explica el menor impacto son las siguientes relaciones: el aumento en la demanda ocasiona un aumento en el precio al mayoreo que se transmite al productor lo que, a su vez, genera un aumento en la producción; dicho aumento ocasiona un descenso en los precios, menor al aumento inicial.

Los cambios en la demanda industrial y en el nivel de precios indican la alta relación entre estas dos variables. El cociente entre los cambios porcentuales de la demanda industrial y los cinco niveles de precios se ubicó en un rango de 2,2 a 7,2, lo cual indica la fuerte influencia que tiene la demanda industrial sobre los diferentes niveles de precios.

La realización de los escenarios 1 y 2 dependerá del compromiso de la industria de la naranja con los demás agentes que participan en la cadena agroalimentaria de la naranja, sobre todo con el productor agrícola. En situaciones de excesos de oferta, la industria deberá comprar los volúmenes que representan un excedente de mercado y que, inevitablemente, ocasionarán una disminución en el precio al productor. Nuevamente, la Secretaría de Agricultura y Desarrollo Rural, Pesca y Alimentación y demás instituciones que tienen relación con la naranja, deberán promover las medidas necesarias para lograr un compromiso con el sector industrial.

El escenario 3 considera la reducción de la superficie de naranja en $5 \%$. La superficie total pasaría de 336 a 319 mil ha, lo cual significaría una disminución de 17000 ha. Por efectos de esta acción y al considerar un rendimiento constante, la producción de naranja en riego y temporal disminuiría en 80 y 160 mil t, respectivamente.

Los cambios en el precio indican que esta variable es muy sensible a los cambios en la superficie sembrada de naranja. Por el cambio señalado, el precio al mayoreo aumentaría de 428 a 614 USD $\$ \mathrm{t}^{-1}$, lo cual representaría un aumento de $43,5 \%$, respecto a la situación base. La contracción de la oferta, vía superficie, sería benéfica para el productor, ya que los precios en riego y temporal se ubicarían en 156 y 130 USD $\$ \mathrm{t}^{-1}$, superiores en 15,2 y 49,1\%, respecto a la situación base. Los resultados anteriores fueron similares a los encontrados por otros autores que han analizado la imposición de medidas que controlan la oferta sobre el ingreso de los productores en cultivos cíclicos. Una reducción del 20 \% en la oferta regional de melón (Cucumis melo L.) en la Comarca Lagunera, región ubicada en el norte de México, aumentaría los precios del melón y las ganancias de los productores de 1051 a $1193 \$ \mathrm{t}^{-1}$ (García-Salazar et al., 2011). En un estudio a nivel nacional sobre el mercado de la sandía (Cucumis sativa L.), la reducción de la producción de esta fruta en los meses en que se observan excesos de oferta aumentaría los precios y la ganancia unitaria de los productores (García-Vázquez et al., 2011).

Los resultados de un estudio indican que los excesos de oferta de papa (Solanum tuberosum L.) en algunos meses, podrían eliminarse a través de la implementación de cambios en la producción de papa de riego; esta política reduciría la volatilidad del precio y aumentarían el ingreso del productor, ya que la planeación de la producción por temporada aumentaría esta variable (García-Salazar et al., 2014). A nivel internacional también se observa la misma 
relación entre los cambios en la superficie y en los precios. Se reporta que una reducción en $10 \%$ en la superficie de maíz en Estados Unidos aumentaría el precio de importación en México en 8,7 \% (González-Rojas et al., 2011).

Los consumidores de naranja saldrían perjudicados, ya que los precios que tendría que pagar serían superiores. El precio al consumidor de naranja en fresco aumentaría de 707 a 830 USD $\$ t^{-1}$, lo que representaría un aumento de $17,4 \%$, en tanto que el precio al consumidor de jugo aumentaría en 18,4\%.

Los cambios en la superficie y en el nivel de precios indican la alta relación entre ambas variables; ante una disminución de $1 \%$ en la superficie, los precios al productor en las zonas de riego y temporal aumentarían en 3,0 y $9,8 \%$, los precios al consumidor de naranja en fresco y jugo en 3,5 y 3,7 \% y, el precio al mayoreo en $8,7 \%$. Estos valores indican el poder que tiene la planeación de la superficie como medida de control de la oferta en el mercado de la naranja en México.

El aumento en los diferentes niveles de precios se debe a la contracción de la oferta, inducida por una acción conjunta de los productores de naranja. Tal acción solo se podría lograr gracias a la organización de los productores de naranja en los distintos estados productores. La Secretaría de Agricultura y Desarrollo Rural, Pesca y Agricultura y otras instancias, como los agentes participantes en el Sistema-Producto de naranja de cada estado, tienen una participación muy importante a través de la instrumentación de acciones que promuevan la planeación de la superficie de naranja.

Desde que México adoptó medidas neoliberales los productores de naranja entraron a mercados de competencia más agresiva, en donde su permanencia dependerá del nivel de organización e integración empresarial. Actualmente, existen esquemas de organización empresarial y que contaron con un amplio menú de opciones jurídicas que permiten cobijar las actividades de los productores rurales (Cedeño y Ponce, 2009).

En otros países la volatilidad de precios también se ha tratado de evitar a través del control de la producción. En el caso de Estados Unidos, diversos estudios indican que el control de la producción de frutas y hortalizas ha permitido aumentar los precios y las ganancias de los productores (Carman y Pick, 1988; Neff \& Plato, 1995; Powers, 1990). El control de la oferta en Estados Unidos para algunas frutas, hortalizas y cultivos especiales se administra a través de las de las órdenes de mercado que pueden ser a nivel federal y estatal. Según reportes del Departamento de Agricultura de Estados Unidos, en la actualidad veinte frutas, hortalizas y cultivos especiales producidos en ese país funcionan bajo el esquema de órdenes de mercadeo (Agricultural Marketing Service, 2014).

Además de la superficie y de la demanda industrial, el aumento en el ingreso de los consumidores ocasionaría un incremento en la demanda de la naranja en fresco, lo que impactaría en el precio al consumidor. Por el lado de la oferta, los factores que podrían aumentar el precio son: condiciones climatológicas adversas como abundantes lluvias que podrían disminuir el nivel de rendimiento, esto disminuiría la disponibilidad de naranja haciéndola más cara en el mercado y el aumento en el precio de la mano de obra (salario medio rural) disminuiría la producción de naranja y esto ocasionaría un aumento en el precio de naranja por la mayor disponibilidad. Es necesario precisar que la influencia en la oferta y demanda es mínima, y/o en ocasiones nula; es por esta razón que no se presenta un cuadro para ilustrar dichos efectos. El comportamiento de la precipitación y de la temperatura (variables que afectan a la oferta) estará en función de las condiciones climáticas prevalecientes en cada una de las regiones productoras de naranja. El salario mínimo rural, otra variable que afecta a la oferta, dependerá de las condiciones laborales existentes en el mercado de trabajo de cada zona productora. El ingreso del consumidor, variable que afecta al consumo, será afectado por el desempeño de la economía nacional. El comportamiento de los precios de los bienes complementarios y sustitutos de la naranja (limón, toronja y mango) será determinado por las condiciones de mercado de cada uno de estos productos. 


\section{Conclusiones}

La formulación de un modelo sintético del mercado de la naranja, que incorpora los factores que afectan a la oferta y a la demanda, se usó para realizar diferentes escenarios que permitirían aumentar los precios al productor de naranja en México, y de esta forma enfrentar la fuerte volatilidad que muestran los precios de esta fruta.

Los resultados de dos escenarios permitieron observar que, por el lado de la demanda, existe una fuerte relación entre las compras de la industria y los precios en sus diferentes niveles, un aumento de $5 \%$ en la demanda industrial de naranja traería un aumento significativo en el precio al productor, lo que significaría un aumento en su ingreso; dichos resultados señalan que la industria procesadora de naranja debe actuar en una situación en la que se presentan excesos de oferta de naranja.

Se requiere el compromiso de la industria para participar en situaciones de sobre oferta a través de la compra de la naranja a precios que beneficien al productor.

Por el lado de la oferta, la elaboración de otros dos escenarios permitió observar que una reducción del $5 \%$ en la superficie sembrada, tendría fuertes impactos sobre los diferentes niveles de precios, lo cual deja de manifiesto el poder que la planeación de esta variable podría tener sobre el mercado de la naranja. En este caso se requiere la organización de los productores para definir la superficie óptima que evite los excesos de la oferta.

Para ordenar el mercado de la naranja, el gobierno debería promover medidas como la planeación de la superficie sembrada y el aumento de la demanda por la industria juguera para mitigar los efectos de volatilidad en los precios.

\section{Referencias}

Agricultural Marketing Service. (2014). Commodities covered by marketing orders. United States Department of Agriculture. https://www.ams.usda.gov/rules-regulations/moa/commodities

Bautista-Mayorga, F. (2017). Planeación de la superficie sembrada y volatilidad de precios en el mercado de la naranja en México [Tesis de Maestría, tesis no publicada]. Colegio de Postgraduados. http://hdl.handle.net/10521/4257

Calderón, M., García, R., López, S., Mora, J. S., \& García, J. A. (2004). Efecto del precio internacional sobre el mercado de la papa en México, 1990-2000. Revista Fitotecnia Mexicana, 27(4), 377-384. https://www.revistafitotecniamexicana. org/documentos/27-4/10a.pdf

Carman, H. F., \& Pick, D. H. (1988). Marketing California-Arizona lemons without marketing order shipments controls. Agribusiness: An International Journal, 4(3), 245-259. https://doi.org/10.1002/1520-6297(198805)4:3<245::AIDAGR2720040304>3.0.CO;2-Y

Cedeño, R., \& Ponce, M. (2009). Organización e integración empresarial de productores rurales. Estudios Agrarios, 40, $111-123$. http://www.pa.gob.mx/publica/rev_40/AN\%C3\%81LISIS/Roberto\%20Cede\%C3\%B10\%20S\%C3\%A1nchez,\%20 Miguel\%20Ponce\%20Gonz\%C3\%A1lez.pdf

Comisión Nacional del Agua. (2017). Temperatura promedio. https://datos.gob.mx/busca/dataset/temperatura-promedio-excel

Comisión Nacional de Salarios Mínimos. (2017). Salario mínimo general promedio de los estados unidos mexicanos 19642016. Secretaría del Trabajo y Previsión Social. https://www.gob.mx/cms/uploads/attachment/file/105354/Salario_ Minimo_General_Promedio_de_los_Estados_Unidos_Mexicanos_1964_-_2016.pdf 
Comité Sistema Productos Cítricos del Estado de Veracruz. (2009). Estudio de mercado para identificación de necesidades de infraestructura logística para la comercialización de jugo de cítricos en Veracruz. https://es.slideshare.net/ cafegourmethelvetia/citricola

Fideicomisos Instituidos en Relación con la Agricultura. (2017). Sistema de costos agrícolas. Resumen de costos. https://www. tamaulipas.gob.mx/campo/wp-content/uploads/sites/40/2019/02/sorgo_gmf_tamaulipas_pv_2017_2017.pdf

Food and Agriculture Organization. (2004). Política de desarrollo agrícola. Conceptos y principios. http://www.fao.org/3/ay5673s.pdf

Food and Agriculture Organization. (2010). La volatilidad de precios en los mercados agrícolas. http://www.fao.org/3/am053s/ am053s00.pdf

Food and Agriculture Organization. (2018). New food balances. http://www.fao.org/faostat/en/\#data/FBS

Francois, J. F., \& Reinert, K. A. (1997). Applied methods for trade policy analysis: A handbook. Cambridge University Press. https://doi.org/10.1017/CBO9781139174824

García, R., García, J. A., \& García, R. C. (2003). Teoría del mercado de productos agrícolas. Colegio de Postgraduados en Ciencias Agrícolas.

García-Salazar, J. A., \& Ramírez-Jaspeado, R. (2013). El tamaño de las unidades de producción de maíz (Zea mays L.): Un desafío para elevar la tasa de utilización de semilla mejorada. Agrociencia, 47(8), 837-849. http://www.scielo.org. $\mathrm{mx} /$ pdf/agro/v47n8/v47n8a8.pdf

García-Salazar, J. A., Skaggs, R. K., \& Crawford, T. L. (2011). Analysis of strategic industry planning and organizacional opportunities for Mexican cantaloupe producers. HortScience, 46(3), 439-444. https://doi.org/10.21273/ HORTSCI.46.3.439

García-Salazar, J. A., Skaggs, R. K., \& Clawford, T. L. (2014). Excess supply and price volatility in the Mexican potato market: A decision making framework. American Journal of Potato Research, 91(3), 291-303. https://doi.org/10.1007/ s12230-013-9349-5

García-Vázquez, A., García-Salazar, J. A., Guzmán-Soria, E., Portillo-Vázquez, M., \& Fortis-Hernández, M. (2011). El mercado de la sandía en México: un estudio de caso sobre excesos de oferta y volatilidad de precios. Región y Sociedad,23(52), 239-260. https://doi.org/10.22198/rys.2011.52.a187

González-Rojas, K., García-Salazar, J. A., Matus-Gardea, J. A., y Martínez-Saldaña, T. (2011). Vulnerabilidad del mercado nacional de maíz (Zea mays L.) ante cambios exógenos internacionales. Agrociencia, 45(6), 733-744. http://www. scielo.org.mx/pdf/agro/v45n6/v45n6a8.pdf

Instituto Nacional de Estadística y Geografía. (2017a). Cuentas nacionales. Producto interno bruto por entidad federativa. https://www.inegi.org.mx/app/indicadores/?tm=0

Instituto de Nacional de Estadística y Geografía. (2017b). Manufacturas. Encuesta mensual de la industria manufacturera (EMIM). https://www.inegi.org.mx/app/indicadores/?tm=0

Instituto Nacional de Estadística y Geografía. (2017c). Precios. https://www.inegi.org.mx/datos/?t=0210

Jaramillo-Villanueva, J. L., \& Benítez-García, E. (2016). Transmisión de precios en el mercado mexinano e internacional de café (Coffea arabica L.): un análisis de cointregración. Agrociencia, 50(7), 931-944. http://www.scielo.org.mx/pdf/ agro/v50n7/1405-3195-agro-50-07-931-en.pdf 
Neff, S. A., \& Plato, G. E. (1995). Federal marketing orders and federal research and promotion programs: background for 1995 farm legislation. United States department of Agriculture. https://www.ers.usda.gov/webdocs/ publications/40612/50840_aer707.pdf?v=2763.8

Powers, N. J. (1990). Federal marketing orders for fruits, vegetables, nuts, and specialty crops. United States department of Agriculture. https://naldc.nal.usda.gov/download/CAT10408634/PDF

Rude, J., Carlberg, J., \& Pellow, S. (2007). Integration to frag $\neg$ mentation: post-BSE Canadian cattle markets, processing capacity, and cattle prices. Canadian Journal of Agricultural Economics, 55(2), 197-216. https://doi.org/10.1111 /j.1744-7976.2007.00088

Secretaría de Medio Ambiente y Recursos Naturales. (2017). Resumenes mensuales de temperaturas y lluvias. https://smn. conagua.gob.mx/es/climatologia/temperaturas-y-lluvias/resumenes-mensuales-de-temperaturas-y-lluvias

Sistema de Información Agroalimentaria y Pesquera. (2017). Anuario estadístico de la producción agrícola. http://infosiap.siap. gob.mx/aagricola_siap_gb/icultivo/index.jsp

Sistema de Información Agroalimentaria y Pesquera. (2018). Consulta por la Tarifa Arancelaria. http://w6.siap.gob.mx/ comercio/AjaxFraccs/conajaxFracc.gobmx.php

Sistema Nacional de Información e Integración de Mercados. (2017). Anuarios estadísticos de mercados nacionales. Frutas y hortalizas. http://www.economia-sniim.gob.mx/SNIIM-AN/estadisticas/e_fyhAnuarioa.asp?

Tomek, W. G., \& Robinson, K. L. (2003). Agricultural product prices. Cornell University Press. https://doi.org/10.1111/14678489.12142

Varian, H. R. (1992). Análisis microeconómico. Antoni Bosh Editor. 